\title{
La formación integral del estudiante y la formación continua de los profesores en la Educación Superior cubana: el papel de la Responsabilidad Social Universitaria (RSU) en su consecución
}

\section{Victoria Ojalvo Mitrany - Laura Curiel Peón}

Universidad de La Habana - Centro de Estudios para el Perfeccionamiento de la Educacion Superior (Cuba)

doi:10.7358/ecps-2015-012-ojal

victoria@cepes.uh.cu lauracp@cepes.uh.cu

INTEGRAL FORMATION OF STUDENT

AND CONTINUOUS FORMATION OF PROFESSORS

IN CUBAN HIGHER EDUCATION: A ROLE

FOR UNIVERSITY SOCIAL RESPONSIBILITY (USR)

LA FORMAZIONE INTEGRALE DEGLI STUDENTI

E LA FORMAZIONE CONTINUA DEI PROFESSORI

NELLEDUCAZIONE SUPERIORE CUBANA: IL RUOLO

DELLA RESPONSABILITÀ SOCIALE UNIVERSITARIA (RSU)

\section{Abstract}

The present article analyzes the main demands facing teachers and students at the present time in the context of higher education in Cuba. These two actors in the educational process should take up their activities with a high sense of responsibility and social commitment to the implications in the social and economic changes that are being developed in the country. Improved training of students and teachers is being investigated worldwide: currently studying University Social Responsibility (URS) are proving their worth in the achievement of such important purposes. In this sense, the Group of Social Interaction University, the Centre of Studies for the Improvement of Higher Education, University of Havana is developing research about training with URS students and professors as a 
contribution to achieve an ideal model of body students and professorate. This training process is designed to promote the social, ethical and emotional for the personal and social welfare.

Keywords: Continuous formation, Cuban Higher Education, Integral formation, Model of student body and professorate, University Social Responsibility.

\section{LA FORMACIÓN INTEGRAL DEL ESTUDIANTE UNIVERSITARIO CUBANO}

¿Cómo considera la educación superior cubana que debe ser el estudiante universitario actual, para que llegue a ser el profesional que el país necesita, a la luz de los importantes cambios que está emprendiendo el país? ¿En qué medida la noción de Responsabilidad Social Universitaria (RSU) puede integrar las distintas características deseadas en estos jóvenes?

Las características del «deber ser» del joven universitario se van conformando a lo largo de su tránsito por la educación primaria y secundaria. En cuanto al nivel terciario de educación, el profesional que emerja de sus aulas, debe formarse a partir del modelo la universidad cubana de hoy:

La universidad ha de ser entendida como un entorno político e intelectual de crucial importancia para la consolidación y fortalecimiento de los valores humanos y de la responsabilidad ciudadana, como la mayor y principal proveedora de oportunidades de aprendizaje y de generación de nuevos conocimientos al más alto nivel científico, capaz de incrementar el impacto social de la actividad de investigación-desarrollo-innovación y extensión que acomete, vinculada a la sociedad, aprendiendo de ella y creciéndose para influir en su perfeccionamiento y transformación. (Diaz-Canel, 2012, p. 10)

H. Hernández Fernández et al. (2013) destacan que la educación superior cubana se sustenta en un modelo de universidad científica, tecnológica y humanista, desde una dimensión dialéctica del desarrollo humano. Entre las características principales de la formación profesional está la formación integral del estudiante, entendida como: «[...] el desarrollo de conocimientos, habilidades, motivos y valores en el estudiante que propician, en su unidad, un desempeño profesional eficiente, ético, responsable y de compromiso con la sociedad cubana» (CEPES, Colectivo de Autores, 2010).

Las bases que sustentan el proceso formativo de la universidad cubana son: el Enfoque Histórico-Cultural de L. S. Vigotsky y sus seguidores, una 
nueva visión del proceso docente como enseñanza-aprendizaje desarrolladores y consecuentemente, de los roles de profesor y estudiante; transformación del papel y las funciones de la institución educativa, que se abre a la comunidad, con vistas al desarrollo de una relación biunívoca entre universidad y sociedad.

La formación integral del estudiante se organiza en distintos niveles: el nivel macro conformado por el plan de estudios; el nivel meso, a través de ciclos, disciplinas, módulos, asignaturas y el nivel micro, con las clases y tareas docentes. En la formación integral se destacan la formación moral y la educación en valores: «[...] una formación humanista centrada en el desarrollo del profesional como ser humano que se construye en el ejercicio de una profesión y como tal se desempeña en ella» (Hernández Fernández et al., 2013, p. 24).

En la formación integral del estudiante interviene el currículo intencionalmente elaborado, integrado, tanto académicamente, como con el mundo real, para que los estudiantes reciban una formación como profesionales, ciudadanos y como individuos; los estudiantes son sujetos activos de su propia formación, desarrollan conocimientos, habilidades y valores, pensamiento analítico y crítico que les permiten resolver problemas profesionales con elevado compromiso social. El papel del profesor no es solo instruir, sino educar y su ejemplo personal juega un papel decisivo en la formación de sus educandos.

El Ministerio de Educación Superior (MES) materializa esta prioridad en el «Enfoque Integral para la Labor Educativa en las Universidades»; en el cual participan todos los centros universitarios del país. El enfoque integral supone la participación de todos los actores de las instituciones educativas: profesores, estudiantes, administrativos, trabajadores, propiciando que la universidad viva un clima de trabajo educativo, con la participación de todas las estructuras universitarias. Este proceso educativo se realiza a través de tres dimensiones: trabajo curricular, de extensión universitaria y las actividades socio-políticas.

Una de las vías para el logro de los altos fines educativos que se proponen las universidades es el Proyecto Integral de Trabajo Educativo (PITE), que consiste en que:

Cada grupo estudiantil elabora, de conjunto con sus profesores, su propio proyecto educativo, y a partir de un diagnóstico de las necesidades educativas individuales y grupales, establecen los objetivos y las acciones que se desarrollarán durante el curso en esta dirección. (Horruitinier, 2007, p. 19)

En el Reglamento Docente y Metodológico. Resolución 210/07 se concreta el perfil deseado del futuro profesional, que es continuación de los fines educativos de los niveles precedentes y se recoge en su primer artículo: 
La formación de los profesionales de nivel superior es el proceso que, de modo consciente y sobre bases científicas, se desarrolla en las instituciones de educación superior para garantizar la preparación integral de los estudiantes universitarios, que se concreta en una sólida formación científico técnica, humanística y de altos valores ideológicos, políticos, éticos y estéticos, con el fin de lograr profesionales revolucionarios, cultos, competentes, independientes y creadores, para que puedan desempeñarse exitosamente en los diversos sectores de la economía y de la sociedad en general. (Ministerio de Educación Superior, 2007)

Es evidente que a lo largo de toda la educación se presentan, como objetivos centrales la formación axiológica de los estudiantes y su compromiso social, la responsabilidad social y ética de los futuros profesionales con la transformación del país. Una carencia en la concepción y desarrollo del trabajo de formación profesional y la formación integral del estudiante universitario cubano es la ausencia de referencias a la formación socio-afectiva, importante objetivo formativo, aspecto esencial tanto para el desarrollo profesional como personal, especialmente valiosa por su condición de base psicológica del desarrollo moral.

A pesar de la conceptualización y operacionalización de la labor educativa y los esfuerzos dedicados a ella en el nivel universitario, todavía no se alcanzan los resultados demandados por nuestra sociedad, por lo que se hace imprescindible continuar perfeccionando esta esfera del trabajo, despojándola del formalismo, conformismo y escasa implicación personal que frecuentemente ahogan sus posibilidades formativas.

En una investigación diagnóstica desarrollada por el Centro de Estudios para el Perfeccionamiento de la Educación Superior (CEPES), se evidenciaron las potencialidades y las limitaciones en la formación integral de grupos de estudiantes de primer año, de distintas facultades universitarias (CEPES, Colectivo de Investigadores, 2010). Este estudio diagnóstico nos permite proyectar acciones formativas para contribuir a mejorar este sensible aspecto de la formación profesional.

Entre las principales potencialidades podemos mencionar la voluntad institucional, intencionalidad y sistema organizado para el desarrollo de la labor educativa en toda la educación superior, como reflejo de la voluntad política del país en la formación integral de nuestros ciudadanos.

Concientización por una buena parte de los docentes de la importancia de la labor educativa en la universidad. La generalidad de los estudiantes reconoce en sus profesores universitarios prestigio académico y científico, lo que los convierte en importante referente en su formación.

En los estudiantes analizados están presentes importantes valores, aunque no siempre ejercen su función regulativa, valoran en alguna medida un cambio favorable en ellos durante su estancia en la Universidad. Un por cien- 
to considerable toma una posición activa ante su propio desarrollo, se sienten identificados con su Facultad, sus profesores y el estudio que realizan, lo que contribuye a su formación integral. Perciben favorablemente la influencia del grupo en su desarrollo, aunque no directamente en sus insuficiencias específicas. En general están identificados con sus raíces cubanas y con los logros de la Revolución.

El estudio realizado identificó algunas limitaciones de las cuales se derivan recomendaciones: No se aprovecha suficientemente todo el potencial formativo que brindan las tres dimensiones de la labor educativa y sus diferentes instancias y niveles, lo que requiere incrementar la preparación psicopedagógica de todos los implicados en la vida universitaria.

Resulta necesario propiciar una mayor participación y protagonismo de los estudiantes en su propia formación, tanto académica como personal, así como en los aspectos organizativos y de gestión de sus facultades.

Es preciso enriquecer y dinamizar la formación axiológica, la participación consciente y la disposición voluntaria en las diferentes tareas formativas.

Tal como hemos visto hasta aquí, el desarrollo de la labor educativa en las universidades cubanas, aunque puede exhibir logros, presenta aún una serie de limitaciones que es preciso abordar desde posiciones científicas. En realidad, no se trata de un problema con límites nacionales, sino que hoy en día ocupa a la educación superior de todo el mundo. Uno de los temas que a nivel internacional está contribuyendo con más fuerza al abordaje de esta temática es el de la Responsabilidad Social Universitaria (RSU)

\section{2. ¿QUÉ ENTENDER POR RESPONSABILIDAD SOCIAL UNIVERSITARIA (RSU)?}

La naturaleza de este concepto es compleja y polémica, por lo que existen variadas posiciones a favor y en contra del mismo, tanto por su origen ajeno al mundo académico, como por sus diversos enfoques e interpretaciones, así como por su aún insuficiente fundamentación teórica. No obstante, esta noción ha despertado gran interés en numerosas universidades de América Latina así como en organizaciones educacionales internacionales. Al respecto, Durat (1999) sostiene que el nuevo paradigma de las instituciones de educación superior es responsabilizar moral y socialmente al joven estudiante para formarlo como universitario, como «Un hombre capaz de asumir sus responsabilidades, de mantener sus compromisos, de pensar por sí mismo, de respetar los sucesos incluso aun cuando vayan en contra de sus pretensiones» (p. 22). 
Ante la diversidad de posiciones y enfoques sobre RSU, en nuestro trabajo adoptamos el enfoque transformador, que implica potenciar el papel de la universidad en la consecución de una sociedad más justa y desarrollada, con una visión ética de su desempeño, que incluye cuatro aspectos esenciales: formación de profesionales, investigación al servicio del mejoramiento social, interacción social y gestión universitaria. Este enfoque supone concebir todo el funcionamiento universitario y todo el quehacer de sus participantes con una visión humanista y ética que contribuya al logro de sus altos fines. Estas características de la RSU están presentes en las concepciones de autores de gran prestigio en el tema como Vallaeys (2005, 2008, 2009), Jiménez (2002, 2008) líder de la red de universidades chilenas «Universidad construye país», así como en representantes de organizaciones educacionales internacionales como la IESALC y la UNESCO.

La preocupación y ocupación de las instituciones de educación superior por contribuir al mejoramiento social no es nueva, sino que acompaña al devenir de la universidad durante siglos. Así lo concibe Hoyos-Vázquez, cuando sostiene que: «La responsabilidad social es de la universidad misma, de su identidad, ya que todo el proceso educativo debe estar orientado a la formación ciudadana, de la cual se sigue todo sentido de responsabilidad en la sociedad» (Hoyos-Vázquez, 2009, p. 427).

La incorporación de este concepto al quehacer universitario brinda una visión holística e integral de las misiones de la institución educativa, con un sustento filosófico y ético, que responde a las nuevas realidades que enfrenta el mundo. La RSU impulsa la toma de conciencia de las universidades sobre su papel en la sociedad, su autoevaluación de como lo hacen y como mejorar su accionar frente a la vulnerabilidad social y económica presentes en nuestras sociedades

Del carácter integrador de la RSU nos hablan Martí y Martí Vilar (2010), al enfatizar que en su formación no sólo hay que enseñar un conjunto de valores propios de una comunidad democrática, sino estructurar el centro y la vida en el aula con procesos en los que la participación en la vida en común, contribuyan a crear los correspondientes hábitos y valores cívicos, favoreciendo un clima óptimo para el desarrollo moral.

Las universidades cubanas, que desde sus orígenes han tenido una vocación ética y de compromiso social, pueden enriquecer su misión y sus resultados a la luz de los aportes de la RSU en la región (Ojalvo, 2014).

$\mathrm{Al}$ analizar la utilidad del concepto de RSU para la educación superior cubana, es preciso considerar nuestras realidades económicas y sociales. Por lo tanto, no podríamos asimilar un modelo de RSU construido para otras realidades, sino que sería preciso elaborar uno sui generis, a la medida de nuestras condiciones, dificultades y posibilidades. Teniendo en cuenta lo an- 
terior, la Educación Superior cubana, que se desarrolla en el seno de una sociedad socialista en pos del logro de la justicia social, cuenta con todas las potencialidades para la consecución de la responsabilidad social de sus instituciones universitarias.

Al tomar en cuenta la amplitud del concepto de RSU, que implica las cuatro funciones sustantivas de la universidad (formación, investigación. extensión y gestión), así como sus interrelaciones, nos focalizamos en este trabajo en el estudio de la formación de estudiantes y profesores universitarios en RSU.

\subsection{Formación de la Responsabilidad Social de los estudiantes}

F. Vallaeys, en su artículo «Breve marco teórico de Responsabilidad Social Universitaria» enfatiza la influencia formativa de la universidad, al afirmar:

La Universidad tiene por supuesto un impacto directo sobre la formación de los jóvenes y profesionales, su manera de entender e interpretar el mundo, comportarse en él y valorar ciertas cosas en su vida ... Influye asimismo sobre la deontología profesional, orienta (de modo consciente o no) la definición de la ética profesional de cada disciplina y su rol social. (Vallaeys, s/f, p. 4)

Los estudiosos del tema afirman que la influencia de la universidad en la formación ética de los estudiantes es un aspecto aún poco tratado en el mundo científico, a pesar de su gran importancia, siendo lo más común que se evalúe el grado de asimilación de contenidos teórico-prácticos de sus carreras y no lo relativo a aspectos del desarrollo de su personalidad. Indudablemente se trata de un objeto de estudio de gran complejidad, al que nos acercamos en la presente investigación.

En investigaciones sobre la formación de la RSU en estudiantes se analizan diferentes variables psicológicas que la integran, Martí Noguera (2011), tales como los comportamientos morales (pro-socialidad, altruismo, acciones de cooperación, participación, auto-control, respeto por las normas sociales); competencias morales, inteligencia social, inteligencia emocional (inter e intra-personal), comunicación, empatía; desarrollo moral evolutivo, formación de valores, autonomía. Aunque existen diversas posiciones al interpretar desde la psicología a la RSU, resulta común que los autores enfaticen los aspectos relativos a la formación ética y de compromiso social, junto con el desarrollo de habilidades o competencias sociales. También la mayoría de los estudiosos consultados destacan el papel de la universidad, en su estrecho vínculo con la sociedad y sus necesidades, como vía esencial para la educación de la RSU en los estudiantes. El interés creciente por el tema no escapa a las organizaciones internacionales. Se intenta cumplir así, un mandato de la UNESCO: 
[...] la educación superior debe no sólo proporcionar competencias sólidas para el mundo de hoy y de mañana, sino contribuir a la formación de una ciudadanía dotada de principios éticos, comprometidos con la construcción de la paz, la defensa de los derechos humanos y los valores de la democracia. (UNESCO, 2009)

A continuación se muestra como distintos autores conciben al estudiante socialmente responsable:

Según Balbo (2008, p. 105) la inclusión de la responsabilidad social universitaria asegura, los aspectos siguientes:

- Formación integral humanística y profesional.

- Formación específica a la ética profesional.

- Reflexión crítica sobre los paradigmas enseńados.

- Apertura social de la enseñanza.

- Orientación de la enseñanza hacia el desarrollo humano sostenible.

- Aprendizaje basado en proyectos sociales.

Buxarrais (2000), afirma que para la educación de la responsabilidad social en los estudiantes resulta esencial su formación axiológica, y propone tres valores fundamentales: la autonomía de las personas; la necesidad de ser personas dialogantes y la tolerancia activa (saber que hay personas que piensan diferente).

$\mathrm{Al}$ analizar el ámbito formativo del enfoque transformador, Gutiérrez Pequeño (s/f) basándose en Martínez (2008), insiste en la importancia de la vinculación del proceso de enseńanza-aprendizaje con la práctica social para el desarrollo de la RSU en los estudiantes, especialmente del llamado aprendizaje-servicio: que consiste en el liderazgo y la participación estudiantil en la solución de problemas de la comunidad, con intencionalidad académica y solidaria, lo que permite la superación de la enseñanza tradicional y la formación de profesionales socialmente responsables.

En el mismo sentido se pronuncia Gaete (2011, p. 117):

Así, los procesos de aprendizaje servicio se transforman en una muy buena instancia para fortalecer la implantación de la RSU, especialmente desde la perspectiva del fortalecimiento de valores y capacidades ciudadanas y sensibilidad social en los miembros de la comunidad universitaria, así como de una mejor valoración e integración de los habitantes de la comunidad local respecto del quehacer universitario.

Hernández (s/f) insiste en la importancia del protagonismo estudiantil para lograr la formación de la RSU mediante el aprendizaje servicio:

El aprendizaje servicio es, por definición, una actividad de los estudiantes. Los protagonistas de las actividades de servicio a la comunidad deben ser los alum- 
nos: si ellos no se apropian del programa, si no lo sienten como algo propio, difícilmente constituya una experiencia de aprendizaje servicio de calidad. (p. 7)

El Equipo Coordinador Universidad Construye País (2006, pp. 43-57) afirma que

Las acciones de la Responsabilidad Social de las Universidades, deben estar orientadas a lograr ciudadanos probos y honestos, que concluyan su carrera profesional con conocimientos que validen las incumbencias para las que les habilitan sus títulos; personas comprometidas con la sociedad de la que forman parte y a la cual volcarán sus conocimientos para mejorar su calidad de vida, tratando de solucionar los problemas que esa sociedad afronta.

Distinguen los siguientes principios y valores que deben guiar a la RSU en las universidades:

- Principios y valores del plano personal: Dignidad de la persona, Libertad, Integridad.

- Principios y valores del plano social: Bien Común y Equidad Social, Desarrollo Sostenible y Medio Ambiente, Sociabilidad y Solidaridad para la Convivencia, Aceptación y Aprecio de la Diversidad, Ciudadanía, Democracia y Participación.

- Principios y valores del plano universitario: Compromiso con la Verdad, Excelencia, Interdependencia y transdisciplinariedad.

Para Navarro Saldaña $(2005$, p. 7) la RSU

es la capacidad y obligación de cada persona, de responder ante la sociedad por acciones u omisiones. Cuando se ejerce, se traduce en compromiso personal con los demás y se refleja en la orientación de las actividades individuales y colectivas en un sentido que contribuyan a generar oportunidades para el desarrollo de las potencialidades y para la satisfacción de necesidades de todos.

Según esta autora para ejercer la responsabilidad social es necesario conocer las necesidades propias y de los demás, desarrollar recursos para responder y optar por las conductas morales que apuntan hacia la supervivencia y desarrollo humano. Enfatiza el vínculo entre responsabilidad social, conducta moral e inteligencia emocional. La conducta moral es entendida como la acción o comportamiento voluntario ejecutado por un ser humano, luego de una decisión en la que intervienen todos los componentes de la moralidad: la habilidad de razonamiento general, el juicio o razonamiento moral, la toma de perspectiva social y los factores afectivos como la empatía y la culpa.

Navarro Saldaña (2005, pp. 11-12) señala que el profesional ejerce su responsabilidad social cuando tiene comportamientos e intenciones como los siguientes: 
- Se preocupa de su propia salud, desarrollo y formación personal y profesional, para ayudar al desarrollo y satisfacción de necesidades de otros.

- Proporciona un servicio profesional de excelencia, tanto por gratificación personal como por dar un servicio adecuado a las necesidades de los demás y lo hace en los servicios o instituciones públicas y privadas.

- Se prepara y perfecciona constantemente para desempeñar bien su profesión y la entiende como un servicio.

- Da respuesta profesional a las necesidades de los destinatarios, sin discriminación por raza, sexo, religión, solvencia económica, etc.

- Participa en equipos de trabajo cooperativo e interdisciplinario para dar una mejor respuesta a las necesidades de la comunidad.

- Participa activamente en la búsqueda de alternativas para disminuir inequidades y contribuir a la equidad social, en lo que compete a su profesión.

- Genera y desarrolla proyectos que contemplan la rentabilidad social, además de la económica.

- Considera las necesidades de la comunidad en el diseño y aplicación de sus proyectos profesionales.

- Investiga en temas de relevancia social, que contribuyen a mejorar la calidad de vida de la población.

- Cuida los recursos naturales, económicos y personales, considerando las necesidades del presente, pero también las de quienes aún no han nacido.

- Realiza acciones profesionales que tienden a fomentar el desarrollo integral y calidad de vida de los seres humanos.

- Actúa con y por la verdad.

Días Sobrinho (s/f) vincula la formación de los estudiantes desde la RSU con la calidad de la educación superior:

La calidad educativa debe estar referida, en última instancia a la formación en su sentido pleno y permanente: intelectual, moral, profesional, social, afectivo y estético. Buscar la calidad en términos de formación corresponde a intentar lograr el mayor desarrollo posible de los sujetos educativos - estudiantes y profesores - en las múltiples dimensiones de la vida humana. [...] La calidad en la educación superior tiene que estar claramente referida a los compromisos de las instituciones con el sentido social de los conocimientos y de la formación, los valores éticos y morales del bienestar colectivo, la democratización del acceso y de la permanencia, la justicia social y el desarrollo sostenible. (p. 108)

F. Vallaeys (s/f) elabora toda una serie de indicadores de RSU relativos a acciones y características que deben estar presentes en todo egresado al concluir su formación profesional: haber participado en cursos de Ética, Responsabilidad Social y Desarrollo; ser sensible a los problemas sociales, universitarios y ambientales y saber qué hacer para participar de su solución. Participar 
en proyectos sociales e investigación-acción en comunidades de aprendizaje. Formar parte de grupos inter-disciplinaros, vivenciar los problemas de pobreza y desigualdad de su país, comprender que es el Desarrollo Humano Sostenible. Entender lo que es el "comercio justo" y ser un "consumidor consciente». Ser capaz de visualizar la utilidad de su profesión en la problemática del desarrollo social de su país. Vincular su especialidad con la solución de los problemas del entorno.

Sánchez Duque y otros (s/f) analizan los trabajos de varios autores, y destacan el vínculo entre RSU y desarrollo de la afectividad, llegando a la conclusión de que los procesos empáticos pueden considerarse la base esencial de la responsabilidad social de un individuo, ya que estas habilidades sociales capacitan al sujeto para interactuar con su ambiente social, interpretar las necesidades y carencias de su entorno, lo ayudan a desarrollar una conciencia más grupal y sentirse afectado por los problemas de los otros. Las limitaciones en la educación socio-afectiva, pueden generar una menor conciencia y orientación hacia lo social, sus problemas y posibles soluciones.

Estos autores citan a otros estudiosos del tema: Martí y Martí Vilar (2010), los cuales en su definición de un estudiante socialmente responsable enfatizan la importancia de las habilidades socio-afectivas en su conformación, ya que lo interpretan como un individuo que es capaz de comprometerse, escuchar y ponerse en el lugar del otro, es decir, un ciudadano empático que se preocupa no sólo por su bienestar sino por el bienestar de todos los que lo rodean.

De la Calle et al. (2007), De la Calle y Jiménez (2011) definen el concepto de Responsabilidad Social del Universitario como el compromiso personal con los demás y con el entorno que asume cada universitario hoy, para el día de mañana ejercer su profesión como un servicio a la sociedad en la búsqueda del bien común. Distinguen las siguientes dimensiones de la Responsabilidad Social Universitaria:

- Compromiso con los demás y con el entorno.

- Descubrimiento personal de los valores.

- Formación de la responsabilidad social.

- Mayor conocimiento de la necesidad del otro.

- Planteamiento del ejercicio profesional desde la búsqueda del bien común. Para Díaz de Aparraguirre la RSU consiste

en promover la generación, desarrollo y difusión del conocimiento en todas sus formas; contribuir a la preservación de la cultura nacional, y desarrollar las actitudes y valores que requiere la formación de personas responsables, con conciencia ética y solidaria, reflexivas, innovadoras, críticas, capaces de mejorar la calidad de vida, consolidar el respeto al medio ambiente, a las instituciones del país y a la vigencia del orden democrático. (Diaz de Aparraguirre, 2009, p. 8, citado por De la Calle et al., 2007) 
Según Mónica Jiménez (2002, p. 95) la RSU se establece:

Formando mujeres y hombres altamente calificados, íntegros e integrales, comprometidos con valores que defienden y difunden activamente; que ven su profesión como una posibilidad de servicio a los demás, y que son capaces de aportar como ciudadanos a la construcción de la sociedad y de responder creativamente a los desafíos de un proyecto país.

Esta autora distingue los siguientes elementos de Responsabilidad Social en los estudiantes (Jiménez, 2002):

- Desarrollar habilidades sociales.

- Desarrollar una relación hacia la sociedad.

- Educar para la conciencia social.

- Crear comunidad.

- Entender la interdependencia global.

- Desarrollar comprensión y habilidades básicas de participación.

- Explorar temas del mundo real.

- Tener oportunidades para la contribución social.

Además de las investigaciones antes mencionadas, que proponen características e indicadores de estudiantes socialmente responsables en general, válidos para la formación en cualquier profesión, existen estudios acerca de la formación en RSU en profesiones específicas. En una investigación realizada por P. Carreño et al. (2006) se promovió la reflexión de destacados profesionales tanto del área farmacéutica como del proyecto RSU, así como de estudiantes y académicos de la Facultad, con la finalidad de que la comunidad interiorizara el concepto de responsabilidad social y que los mismos estudiantes plantearan, desde su perspectiva, el rol de los Químicos Farmacéuticos en la sociedad. El resultado de este trabajo fue la delimitación de las siguientes características de un estudiante universitario de Química Farmacéutica responsable:

- Se preocupa de su autoconocimiento, bienestar físico, psíquico y emocional para su desarrollo integral.

- Comprende que todas sus acciones repercuten en su entorno.

- Es un ser consciente que como persona y como profesional del área de la salud está al servicio de la sociedad.

- Sus actividades se orientan a mejorar la calidad de vida de todas las personas.

- Se mantiene actualizado para un mejor desempeño profesional.

- Se comporta de manera ética.

- Trabaja en forma cooperativa, en equipos multidisciplinarios.

- Investiga y participa en el desarrollo de temas con beneficio social, ecológico y cultural. 
- Trabaja en forma empática, asertiva, creativa y proactiva.

- Ejerce liderazgo participativo.

Para Zolezzi (s/f, p. 258) de la Pontificia Universidad Católica del Perú, el perfil del egresado de Derecho que postula la responsabilidad social universitaria es el siguiente:

- Un estudiante preocupado por las injusticias a su alrededor y con voluntad de comprometerse en acciones concretas.

- Un estudiante que haya podido desarrollar su propia capacidad solidaria en acciones de voluntariado conducidas desde la Universidad.

- Un estudiante informado, capaz de contextualizar su saber especializado en vista a la solución de los problemas cruciales de su sociedad.

- Un estudiante capaz de escuchar, intercambiar y entrar en empatía con el otro, es decir que haya podido beneficiar de experiencias sociales formativas a nivel emocional. Un estudiante formado a la ética del diálogo.

- Un estudiante promotor de democracia y participación, que sabe ser ciudadano, es decir que «sabe gobernar y ser gobernado».

A partir del análisis de las diferentes concepciones e indicadores aquí mostrados, podemos generalizar los aspectos que caracterizan a un estudiante socialmente responsable:

- Desarrollo integral, de todas las potencialidades físicas y mentales en función no solo personal, sino también social, por su contribución a la calidad de vida, la supervivencia, el respeto al medio-ambiente y el desarrollo humano sostenible.

- Participación y compromiso con la transformación social, el logro de la justicia social, la equidad y el desarrollo. Vivenciar y desarrollar la sensibilidad ante los problemas sociales y sus posibles soluciones desde la profesión propia.

- Formación moral: presencia de valores tales como la responsabilidad, (entendida como responder tanto por lo que se hace como por las omisiones), honestidad, solidaridad, conciencia social, la equidad.

- Desarrollo de las bases socio-afectivas de la moralidad: empatía, habilidades sociales y comunicativas.

- Ejercicio de la profesión en función del mejoramiento social: servicio profesional de excelencia, auto-perfeccionamiento constante, cooperación, trabajo en equipo, interdisciplinariedad. Cuidado de los recursos naturales, económicos y humanos. Ser reflexivo, crítico, innovador en el desempeño de la profesión.

Al comparar estas características con el perfil deseado del estudiante universitario cubano encontramos gran coincidencia en una serie de aspectos tales como la formación integral: científico-técnica, humanística y axiológica, en función de la participación y el compromiso para el mejoramiento y la transformación social; desempeño ético y responsable de su profesión, auto-perfec- 
cionamiento y capacitación constante. No existe coincidencia en los aspectos relativos a la formación socio-afectiva: habilidades sociales y comunicativas, empatía, asertividad, liderazgo estudiantil, los cuales no se mencionan explícitamente en los documentos normativos de la Educación Superior cubana.

\subsection{El «deber ser» del Docente universitario y su formación en Responsabilidad Social Universitaria}

Los avances de la ciencia y la tecnología constituyen signos característicos de la sociedad contemporánea. Los cambios generados en la vida cotidiana y en la producción de bienes han transformado la convivencia y las relaciones de poder, retando las costumbres, la moralidad y la vida misma. Han surgido nuevos campos de estudio de la ciencia y la ética como son la bioética y los estudios sociales de la ciencia y la tecnología (Delgado, 2014).

Todas estas transformaciones han suscitado dilemas éticos que involucran tanto a investigadores como a actores sociales y organizaciones internacionales.

En este sentido, la educación debe promover una capacitación crítica y contextualizada que contribuya a la formación de ciudadanos que se impliquen en la vida pública y por tanto en los debates éticos relacionados con las tecno-ciencias, propiciando de esta manera una mayor inclusión social (Albornoz \& López, 2010).

En la Declaración de la Cumbre de Jefes de Estado y de Gobierno, que tuvo lugar en el año 2006, la educación superior se concibe como un ámbito que debe estar centrado en la formación, la transmisión del conocimiento y la contribución a la generación de una ciudadanía responsable (Albornoz \& López, 2010).

En la Declaración de la Conferencia Regional de Educación Superior en América Latina y el Caribe (CRES, 2008) se expresa: «Reivindicamos el carácter humanista de la Educación Superior, en función del cual ella debe estar orientada a la formación integral de personas, ciudadanos y profesionales, capaces de abordar con responsabilidad ética, social y ambiental los múltiples retos implicados en el desarrollo endógeno y la integración de nuestros países, y participar activa, crítica y constructivamente en la sociedad" (IESALC UNESCO, 2008, p. 5). Se legitima de esta manera el papel de la Educación Superior en lo referido a la responsabilidad social y se hace un llamado a generar acciones en esta dirección.

En consonancia con esta idea, la autora T. Gómez (2011) expresa que la responsabilidad social en la educación se concibe como una forma de hacer educación, el modo de ser una institución educativa y su proyección ética. Detrás de esta concepción subyace un concepto de sociedad y de relación entre es- 
ta y las instituciones educativas, así como, una concepción de la función social de la educación. Cualquier institución educativa interesada en ser socialmente responsable debe tener presente cuál es el impacto que tienen sus actividades en tres planos: en los estudiantes, en los trabajadores (personal académico y no académico) y en el entorno (social, ambiental, cultural, político y económico).

El papel de los docentes resulta fundamental al tener la responsabilidad de formar profesionales competentes en el ejercicio de su profesión, conscientes de que ser universitario no sólo implica un trabajo bien remunerado, sino un compromiso de poner su conocimiento en función de la sociedad (Alfonso, 2011).

La responsabilidad social del profesor en su función docente, implica el compromiso para desarrollar estrategias relacionadas con su formación académica y actividades para gestionar el conocimiento en aras de lograr la formación del profesional requerido por la sociedad. Le corresponde promover la participación del estudiante en la construcción del conocimiento, por lo cual debe dominar los propósitos institucionales, concebir científicamente la formación integral del estudiante y reflexionar sobre la práctica y los resultados (Vera et al., 2012).

En opinión de Arredondo (2009) los docentes son protagonistas y líderes en el marco de la universidad, por lo cual deben participar como autores de las políticas educativas y en el mejoramiento continuo de la malla curricular. Esta participación debe ser a través de la reflexión e inquietud ética y epistemológica desde la especialidad y en articulación con el enfoque RSU.

En el Comunicado Final de la Conferencia Mundial de Educación Superior (2009), se hace un llamado a «establecer un alto nivel en la formación de los docentes [...] mediante planes de estudio que les brinden herramientas idóneas para educar a los estudiantes como ciudadanos responsables» (UNESCO, 2009, p. 8).

Los elementos vistos con anterioridad sirven como premisa para el análisis de cuáles deben ser las características de los docentes universitarios en la actualidad, cuáles son los retos que enfrentan en su ejercicio profesional y hacia dónde debe apuntar su formación.

M. Segura (2004) plantea que uno de los factores centrales del cambio educativo siguen siendo los docentes. Su formación y actualización debe apuntar a un docente que posea una visión general de las situaciones, con amplitud de pensamiento, que se destaque por su ética y sea modelo para sus estudiantes.

Esta autora expresa que el nuevo orden educativo constituye un reto para los docentes de la Universidad de Carabobo (donde labora) y señala un grupo de competencias que deberán poseer: conocimiento y destrezas andragógicas; alto nivel de competencias; conocimiento y dominio de las tecnolo- 
gías de la información y comunicación; altos estándares de calidad; destrezas gerenciales e innovación y creatividad.

Por su parte, Z. Bozu y P. J. Canto (2009) destacan que los docentes deben ser capaces de promover el desarrollo de competencias para que los sujetos puedan continuar aprendiendo a lo largo de su vida y se desempeñen de manera satisfactoria y pertinente en un mundo cambiante y complejo, de ahí la importancia de la definición de un nuevo perfil docente.

Estos autores mencionan un grupo de competencias básicas que deben poseer los docentes universitarios, de acuerdo a las características del Espacio Europeo de Educación Superior:

- Competencias cognitivas específicas a una determinada disciplina.

- Competencias metacognitivas, propias de un profesional reflexivo y crítico con su propia enseñanza y práctica docente.

- Competencias comunicativas.

- Competencias gerenciales.

- Competencias sociales que le permitan acciones de liderazgo, de cooperación, de trabajo en equipo.

- Competencias afectivas (motivaciones, actitudes, conductas) que propicien el desarrollo de una docencia responsable y comprometida con el logro de los objetivos formativos.

A su vez expresan la necesidad de que los docentes posean un grupo de competencias pedagógicas y didáctico-metodológicas para lo cual retoman las planteadas por Valcárcel (2003):

- Conocimiento del proceso de aprendizaje del estudiante en contextos académicos y naturales.

- Planificación de la enseñanza y de la interacción didáctica.

- Utilización de métodos y técnicas didácticas pertinentes.

- Gestión de interacción didáctica y de las relaciones con los alumnos.

- Evaluación, control y regulación de la propia docencia y del aprendizaje.

- Gestión de su propio desarrollo profesional como docente.

En opinión de M. Del Moral y L. Villalustre (2004), para hacer frente a los nuevos requerimientos pedagógicos, los docentes deberán poseer un conjunto de competencias instrumentales, interpersonales y sistémicas.

- Competencias instrumentales:

- Capacidad para facilitar el aprendizaje.

- Habilidad para diseñar materiales autoformativos que permitan la contextualización tanto de los contenidos como de las actividades integradas en el entorno virtual.

- Capacidad para la gestión y organización de la estructura y dinámica interna de las actividades formativas.

- Capacidad para evaluar los aprendizajes. 
- Competencias interpersonales:

- Capacidad para generar espacios de intercomunicación en el entorno virtual para facilitar el intercambio de información y permitir una fluida y ágil comunicación entre los distintos miembros de la comunidad virtual de aprendizaje integrada por docentes y estudiantes.

- Habilidad para promover la participación activa de los estudiantes en la construcción de sus propios conocimientos.

- Habilidad para crear entornos de trabajo colaborativo, en tanto dinamizadores de grupos de trabajo, adquiriendo un compromiso mutuo compartido con el aprendizaje de los estudiantes. Organizadores y planificadores de las actividades grupales a desarrollar, con el fin de que se orienten a la consecución de unos objetivos previamente determinados.

- Competencias sistémicas:

- Capacidad para motivar el proceso de auto-aprendizaje a partir del diseńo y desarrollo de proyectos colaborativos.

- Capacidad para asesorar y orientar el proceso individual del aprendizaje, atendiendo a la diversidad del alumnado y de los contextos en los que tiene lugar el proceso de enseñanza-aprendizaje.

En este sentido, Mas (2011) plantea seis competencias básicas de la función docente:

- Diseñar la guía docente de acuerdo con las necesidades, el contexto y el perfil profesional, todo ello en coordinación con otros profesionales.

- Desarrollar el proceso de enseñanza aprendizaje propiciando oportunidades de aprendizaje tanto individual como grupal.

- Tutorizar el proceso de aprendizaje del alumno propiciando acciones que le permitan una mayor autonomía.

- Evaluar el proceso de enseñanza-aprendizaje.

- Contribuir activamente a la mejora de la docencia.

- Participar activamente en la dinámica académico organizativa de la institución (Universidad, Facultad, Área, Departamento, titulaciones ...).

Más allá de las clasificaciones que proponen los diferentes autores, pueden apreciarse varios puntos de coincidencia entre ellos, los cuales pueden dar cuenta de elementos que pudieran integrar el perfil del docente universitario, de acuerdo a las exigencias actuales de la Educación Superior:

- Dominio de los contenidos de la especialidad en la cual son formados.

- Dominio de herramientas pedagógicas y andragógicas que permitan guiar de manera intencionada y científica el Proceso de Enseñanza Aprendizaje (PEA).

- Concepción del estudiante como sujeto activo en el marco del PEA.

- Concepción de una formación profesional integral que abarque los aspectos éticos y el compromiso social de los graduados. 
- Dominio de las tecnologías de la información y las comunicaciones.

- Dominio de herramientas de la gestión.

- Habilidades socioafectivas y comunicativas.

- Preocupación por la formación permanente.

¿Están presentes estos elementos en la formación y desarrollo de los docentes universitarios en nuestro país?

A continuación se analizan los principales criterios, documentos normativos e investigaciones que apuntan al «deber ser» del docente universitario cubano, para contrastar esta información con los resultados de estudios acerca de su desempeño docente.

En Cuba, la formación y desarrollo del claustro universitario es objeto de interés del Ministerio de Educación Superior (MES) de ahí que se haya definido el Área de Resultados Clave (ARC) \# 2 «Claustro revolucionario de excelencia» donde se recogen objetivos de trabajo dirigidos al incremento de la formación integral de los docentes, enfatizándose la preparación político ideológica, pedagógica y científica.

Alarcón, actual Ministro de Educación Superior en Cuba (2013, citado por Sandó et al., 2013, p. 93) plantea: «[...] no basta con la excelencia de los planes y programas de estudio, ni con la magnificencia de las instalaciones o ambientes; a nuestro juicio, este empeño, requiere de docentes comprometidos y con una alta preparación, pues una buena parte de la capacidad de influencia que ejerce el profesor en los estudiantes se relaciona primero con lo que él es como persona, ya que el maestro instruye con lo que sabe, pero educa con lo que es». Más adelante señala que la formación de docentes: «responde a un perfil en función de formar y consolidar valores patrios y un profundo sentido humanista, al mismo tiempo que preserva, genera y promueve conocimientos, habilidades y valores que se reflejan en la formación integral del profesional».

En el Capítulo II del Reglamento para la aplicación de las categorías docentes de la educación superior (MES, 2006) se señalan como funciones generales del personal docente universitario:

- Educar para la formación de valores éticos y morales, convicciones personales, hábitos de conducta y personalidades integralmente desarrolladas que piensen y actúen creadoramente, aptas para construir la nueva sociedad y defender las conquistas de la Revolución.

- Realizar la planificación, ejecución y control del proceso docente de pregrado y postgrado en todas sus formas, de acuerdo con su categoría docente.

- Desarrollar actividades metodológicas y de superación inherentes al proceso docente de pregrado y postgrado, hasta el nivel de actualización que requiera el desarrollo exitoso de las funciones correspondientes a su categoría. 
- Elevar constantemente sus conocimientos pedagógicos, científico-técnicos y culturales.

- Realizar investigaciones, trabajos de desarrollo y de innovación tecnológica, así como servicios científico técnicos y de aplicación que contribuyan al desarrollo de las fuerzas productivas de la sociedad y al perfeccionamiento de la vida social en su conjunto.

- Cumplir las regulaciones establecidas para el personal docente universitario.

En espacios posteriores de este reglamento, se especifican las funciones propias de cada categoría docente en particular.

Los jóvenes recién graduados que se inician como profesores noveles (adiestrados) cuentan también con posibilidades de formación docente. Según el Informe de Balance del Trabajo emitido por el MES en enero del 2012, en la actualidad los Centros de Educación Superior (CES) tienen implementadas estrategias para la preparación y superación de los adiestrados en este sentido (MES, 2012).

La Universidad de La Habana cuenta con el Plan de Desarrollo para jóvenes profesionales que se encuentran en adiestramiento laboral, el cual se ha diseñado como parte de la política que posee la institución para la atención a los adiestrados. Este posee un carácter abierto y atiende a la proyección de la institución y a la caracterización de los jóvenes profesionales. Para su proyección e implementación cuenta con los componentes: superación e investigación, docencia, ejercicio de la especialidad y un componente transversal de formación integral político-ideológica. El Plan se prevé con una duración de tres años (Universidad de La Habana, 2010). En este documento puede apreciarse que: si bien se incluyen acciones relacionadas con la Educación Superior y sus tendencias, la formación psicopedagógica se concibe descentralizada y no se trabajan aspectos de relevante importancia como la formación socio-afectiva y emocional del docente, ya que sólo existe una propuesta vinculada con estos temas (una de las 3 asignaturas de la Maestría de Psicología Educativa) donde se tratan algunos contenidos vinculados con la Comunicación en el espacio de clases. Su elección es optativa por lo que este conocimiento llega sólo a una parte de esos profesores.

Como complemento de las normativas establecidas, se realizan investigaciones para contribuir a la determinación del perfil del profesor universitario cubano.

G. Suárez et al. (2012) muestran resultados de investigación relacionados con la formación y desarrollo basado en competencias de docentes en la Filial Universitaria de Aguada de Pasajeros, provincia Cienfuegos.

Las competencias determinadas en el marco de dicha investigación fueron: 
- Competencias básicas:

- Capacidad de interpretación.

- Capacidad de expresión oral.

- Capacidad de escuchar.

- Capacidad de comunicación.

- Capacidad de expresión escrita.

- Capacidad de lectura.

- Competencias genéricas:

- Capacidad de educar e instruir.

- Capacidad para desarrollar los valores e ideología de la Revolución.

- Capacidad de aprendizaje continuo desde diversas fuentes.

- Pensamiento crítico y reflexivo.

- Capacidad de autodesarrollo.

- Autoconfianza para asumir retos y riesgos.

- Competencias especificas:

- Dominio de los contenidos propios de la asignatura.

- Capacidad de organizar, planificar y controlar el proceso docente educativo.

- Dominio del uso de los medios que se utilizan en el programa, el libro, las guías y las TIC.

- Dominio del trabajo metodológico, investigativo y extensionista.

- Capacidad de orientar y controlar el trabajo independiente de los estudiantes.

$\mathrm{Al}$ analizar los componentes estructurales de las actividades básicas generalizadas del profesor en las sedes universitarias municipales, Ortiz et al. (2013) señalan:

- Actividades básicas generalizadas y acciones:

- Planificar actividades docentes: elaborar objetivos de la clase, organizar los contenidos esenciales a tratar; elaborar las tareas docentes; determinar los métodos de enseńanza aprendizaje; seleccionar y/o elaborar los medios de enseńanza-aprendizaje; establecer los criterios de evaluación de la clase y elaborar los instrumentos correspondientes; elaborar las orientaciones del estudio independiente de los estudiantes.

- Ejecutar y controlar las actividades docentes: controlar, corregir y retroalimentar a los estudiantes sobre la ejecución del estudio independiente; motivar al estudiante; presentar y discutir los objetivos de la clase; establecer la Base Orientadora de la Acción conjuntamente con los estudiantes; orientar el nuevo contenido y la tarea docente a ejecutar; controlar y corregir la ejecución de la tarea docente; aplicar los instrumentos de evaluación; orientar el estudio independiente y la bibliografía a consultar. 
Otras acciones que se destacan son: caracterizar a los estudiantes individual y grupalmente; atender necesidades educativas individuales y grupales; aplicar y calificar exámenes finales; tutorar trabajos de investigación estudiantil y trabajos de diploma; organizar y ejecutar actividades de extensión universitaria con la participación de estudiantes y realizar trabajo científico metodológico.

Cabría preguntarse ¿cómo se expresan los aspectos pautados en la práctica universitaria? Ojalvo (2012) analiza algunos resultados obtenidos en el estudio de la figura del docente y su contribución a la formación integral de los estudiantes universitarios, en el marco de la investigación: «Estrategia educativa para la formación integral de estudiantes de segundo año de la Universidad de La Habana».

Como potencialidades para el trabajo educativo se enfatiza que la mayoría de los investigados está presente la motivación por la profesión docente, la comprensión de sus principales funciones, haber recibido preparación sistemática y sentir satisfacción por el desempeño del trabajo docente educativo. Sin embargo, entre las limitaciones señaladas se encuentran las incomprensiones por una parte de los docentes de la necesidad de recibir capacitación especial para llevar a cabo el trabajo formativo y la insuficiente preparación para desarrollar la evaluación integral de los jóvenes.

Si bien los elementos vistos hasta el momento no agotan el tema de la formación y desarrollo del claustro de nuestras universidades, sirven como premisa para la reflexión y arrojan algunas pistas de lo que pudieran denominarse fortalezas y limitaciones con vistas al acercamiento o no a las exigencias actuales de la educación superior.

- Fortalezas:

- La formación y desarrollo del claustro universitario constituye una voluntad institucional, como expresión del proyecto social cubano.

- En todas las instituciones universitarias del país se tienen concebidas acciones tanto para la formación inicial de docentes como para la formación continua, en aspectos tanto de las especialidades como psicopedagógicos y político-ideológicos.

- El claustro universitario goza de prestigio académico ante los estudiantes, avalado por su experiencia en la docencia y la investigación científica.

- Limitaciones:

- Debe fortalecerse la formación psicopedagógica en aspectos socioafectivos y en estrategias formativas de manera que los docentes posean herramientas útiles para realizar el trabajo educativo con los estudiantes y de conjunto con la formación en aspectos como la didáctica, se contribuya a guiar de forma intencionada y científica el PEA.

Los elementos tratados arriba dan cuenta de la importancia y la necesidad en la actualidad de la formación psicopedagógica de nuestros docentes 
universitarios, especialmente en el ámbito socio-afectivo, por la necesidad de la formación ética, socialmente responsable y afectiva que se demanda y que debe traducirse en la formación de profesionales más integrales.

El análisis realizado nos evidencia lo complejo que resulta el logro de este «deber ser» del estudiante y del profesor universitario. Es una tarea que requiere de una preparación especial de todos los implicados, sobre bases científicas, la sinergia de todas las funciones y actores universitarios, sobre la base de un liderazgo ético de la universidad. A los efectos investigativos resulta inabarcable toda la complejidad de este objeto de estudio, por lo que nos planteamos tareas parciales que vayan contribuyendo a enriquecer la comprensión del mismo y nos permitan acercarnos desde la ciencia, al modelo de formación del estudiante y del docente cubano desde la RSU, teniendo en cuenta nuestras posibilidades reales y condiciones histórico-concretas.

Abordaremos en nuestra investigación dos grandes áreas de la formación del estudiante y del profesor en RSU: (1) formación ética, y (2) formación socio-afectiva, que incluye el desarrollo de habilidades sociales y de comunicación.

La definición que asumimos en nuestro estudio es la siguiente:

La formación de profesores y estudiantes en Responsabilidad Social Universitaria se entiende como el proceso y resultados educativos para la contribución al desarrollo profesional y personal de profesores y estudiantes de carácter humanista, ético y socio-afectivo, en las condiciones histórico-concretas de nuestra sociedad, a partir del modelo de universidad cubana al cual aspiramos, que promueva la participación y el compromiso con la transformación social, facilite la convivencia y el bienestar personal y social.

La formación soco-afectiva se entiende como el proceso educativo intencionado, sistemático y permanente cuyo objetivo es potenciar el desarrollo social, ético y emocional, a partir de la interacción social, en el marco de una cultura y valores deseados, como expresión de la unidad de lo cognitivo, afectivo y valorativo del desarrollo de la personalidad integral y auto-determinada, para facilitar la convivencia, el bienestar personal y social.

\section{BIBLIOGRAFÍA}

Albornoz, M., \& López, J. A. (2010). Presentación. In M. Albornoz \& C. López (Coords.), Ciencia, tecnología y universidad en Iberoamérica. Colección Metas Educativas 2021. Retrieved from: http://www.oei.es/cienciayuniversidad/spip. php?article2337 (consulted 25/03/2014). 
Alfonso, E. (2011). Responsabilidad del docente universitario. Didac Nueva Ëpoca, 58, 36-40. Retrieved from: http://www.uia.mx/web/files/didac/58.pdf (consulted 25/03/2014).

Arredondo, A. (2009). Responsabilidad social aplicada al ámbito universitario. Retrieved from: http://www.ucsm.edu.pe/moodledata/portaljoomla/images/ programas/File/Modulo\%203\%20pdf.pdf (consulted 25/03/2014).

Balbo, Josefina (2008). La enseñanza de la ética a través de la inclusión de la responsabilidad social universitaria en el currículo. Revista Educación en Valores. Universidad de Carabobo, 1(9), Enero-Junio, 99-107.

Bozu, Z., \& Canto, P. J. (2009). El profesorado universitario en la sociedad del conocimiento: competencias profesionales docentes. Revista de Formación e Innovación Educativa Universitaria, 2(2), 87-97. Retrieved from: http:// tecnologiaedu.us.es/mec2011/htm/mas/3/31/26.pdf (consulted 25/03/2014).

Buxarrais, R. (2000). Características de los centros educativos para una educación en valores. Conferencia dictada en las XIX Jornadas Interdisciplinares: La Responsabilidad Social en la Educación, Diciembre 2000. http://www.ua-ambit. org/jornades2000/Ponencias/j00-buxarrais.htm (consulted 25/03/2014).

Carreño, P., Bonilla, S., Rubio, C., Cortés, M., \& Ojeda, J. (2006). Responsabilidad Social en la formación del Químico-farmacéutico de la Universidad de Valparaiso. Facultad de Farmacia, Universidad de Valparaíso, Valparaíso, Chile. Edusfarm, Revista d'Educació Superior en Farmàcia, 2(2), 2007, 1-25.

CEPES, Colectivo de Investigadores. 2010. Estrategia educativa para la formación integral de estudiantes de 2do. año de la Universidad de La Habana. Informe parcial de investigación: Etapa diagnóstica. La Habana: Inédito.

De La Calle, C., García Ramos, J. M., Jiménez Armentía, P. (2007). La formación de la responsabilidad social en la Universidad. Revista de Educación de la Universidad Complutense de Madrid, 18(2), 47-66.

De la Calle, C., \& Jiménez Armentía, P. (2011). Aproximación al concepto de Responsabilidad Social del Universitario. Revista Comunicación y Hombre, 7, 236-247. Retrieved from: http://www.comunicacionyhombre.com/articulo. php?articulo=108 (consulted 11/10/2013).

Delgado, C. J. (2013). Ciencia, tecnología y ciudadanía: cambios fundamentales y desafíos éticos. Revista Universidad de La Habana, 276, 34-47.

Del Moral, M., \& Villalustre, L. (2004). Hacia un nuevo perfil del docente universitario: exigencias formativas para la innovación tecnológica. Retrieved from: http://edutec2004.lmi.ub.es/pdf/59.pdf (consulted 25/03/2014).

Dias Sobrinho, J. (s/f). Calidad, pertinencia y responsabilidad social en la universidad latinoamericana y caribeña. http://www.iesalc.unesco.org.ve (consulted 11/10/2013).

Diaz-Canel Bermúdez, M. (2012). Conferencia inaugural Evento Internacional Universidad 2012, La Habana, Palacio de Convenciones. 
Durat, J. M. (1999). La Organización ética de la escuela y la transformación de valores. Barcelona: Paídos.

Equipo Coordinador UPC (2006). Responsabilidad Social Universitaria, una manera de ser Universidad. Teoría y práctica en la experiencia chilena. Santiago de Chile: Construye País.

Gaete Quezada, R. (2011). La responsabilidad social universitaria como desafío para la gestión estratégica de la Educación Superior: el caso de Espańa. Revista de Educación, 355, Mayo-Agosto, 109-133.

Gómez, T. (2011). Una palabra de la editora: responsabilidad social en la educación. Didac Nueva Época, 58, 2-3. Retrieved from: http://www.uia.mx/web/files/ didac/58.pdf (consulted 25/032014).

Gutiérrez Pequeño, J. M. (s/f). Responsabilidad Social Universitaria: una nueva mirada a la relación de la universidad con la sociedad desde la perspectiva de las partes interesadas. Un estudio de caso. Tesis de Doctorado, Universidad de Valladolid.

Hernández Fernández, H., Hernández, A., \& González, M. (2013). La formación profesional en el contexto de la educación superior cubana. Características principales. In A. Hernández (Coord.), Aportes de Enfoque histórico-cultural a la educación. Experiencias de su aplicación en la Universidad de La Habana, Cuba. Editorial FEDUN.

Hernández, A. M. (s/f). Aprendizaje servicio como elemento para el fortalecimiento de la calidad académica. Revista Semestral Dialogus. Universidad Valle del Momboy.

Horruitiner Silva, P. (2007). El proceso de formación. Sus características. Revista Pedagogía Universitaria, 12(4), 13-48.

Hoyos-Vázquez, G. (2009). Educación para un nuevo humanismo. Magis, Revista Internacional de Investigación en Educación, 2, 425-433.

IESALC - UNESCO (2008). Declaración de la Conferencia regional de Educación Superior en América Latina y el Caribe. Retrieved from: http:/www.sisbi.uba. ar/novedades/DeclaracionCRES2008.pdf (consulted 11/10/2013).

Jiménez, M. (2002). Marco conceptual sobre la Responsabilidad Social Universitaria. Documento preparado por Equipo Coordinador "Universidad: Construye Pais» y Académicos de las universidades aliados al Proyecto.

Maldonado Calleja, N. et al. (2011). Congreso de transformación educativa. Responsabilidad Social Universitaria, un punto de vista de los estudiantes Universidad Autónoma del Estado de México.

Martí Noguera, J. J. (2011). Responsabilidad Social Universitaria. Estudio acerca de los comportamientos, los valores, y la empatía en estudiantes de universidades iberoamericanas. Tesis de Doctorado, Universidad de Valencia.

Martí-Vilar, M. et al. (2011). Responsabilidad Social Universitaria: estudio iberoamericano sobre influencia de la educación en la formación de profesionales responsables con la sociedad. Retrieved from: http://www.cyta.com.ar/ta1003/v10n3a1. htm (consulted 12/10/2012). 
Mas, O. (2011). El profesor universitario: sus competencias y formación. Revista de Currículum y Formación del Profesorado, 5(3), 195-211. Retrieved from: http://www.ugr.es/ recfpro/rev153COL1.pdf (consulted 25/03/2014).

Ministerio de Educación Superior (MES) (2006). Reglamento para la aplicación de las categorias docentes de la Educación Superior.

Ministerio de Educación Superior (MES) (2007). Reglamento docente metodológico publicado en cultura docente.

Ministerio de Educación Superior (MES) (2012). Informe de balance de trabajo.

Navarro Saldaña, G. (2005). Comportamiento socialmente responsable. Universidad de Concepción (Chile).

Ojalvo, V. (2012) ¿Está capacitado el profesor universitario para la formación integral del estudiante? Ponencia presentada en el Congreso Internacional Universidad 2012, La Habana (Cuba).

Ojalvo Mitrany, V. (2014). Carácter polémico del concepto de Responsabilidad Social Universitaria. ¿Es útil a la Educación Superior cubana? Ponencia presentada en el Evento Internacional Universidad 2014, la Habana (Cuba).

Ortiz, T., Hernández, A., \& Rodríguez, A. G. (2013). El método teórico del análisis estructural de la actividad: su aplicación en el estudio de la actividad profesional del profesor universitario de las Sedes Universitarias Municipales. In A. Hernández (Coord.), Aportes de Enfoque histórico-cultural a la educación. Experiencias de su aplicación en la Universidad de La Habana, Cuba. Editorial FEDUN

Sánchez Duque, J. W., Puerta Lopera, I. C., \& Aragón Tabón, O. E. (s/f). Responsabilidad Social Universitaria en la Fundación Universitaria Luis Amigó (FUNLAM) Medellin, Colombia.

Sandó, P. et al. (2013). La experiencia cubana en la formación del profesor universitario. Revista de Docencia Universitaria. REDU, Número monográfico dedicado a Formación docente del profesorado universitario, 11(3), Octubre-Diciembre, 91-123. Retrieved from: http://www.red-u.net/ (consulted 22/04/2014).

Segura, M. (2004). Hacia un perfil del docente universitario. Revista Ciencias de la Educación, año 4, 1(23), 9-28. Retrieved from: http://servicio.bc.uc.edu.ve/ educacion/revista/a4n23/23-1.pdf (consulted 25/03/2014).

Suárez, G., Iglesias, M., \& Basulto, B. (2012). La formación y desarrollo basado en competencias de los profesores universitarios en la Filial de Aguada de Pasajeros. Pedagogía Universitaria, 17(2), 154-163. Retrieved from: http://cvi.mes. edu.cu/peduniv/index.php/peduniv/article/view/24 (consulted 25/03/2014).

UNESCO (2009). La nueva dinámica de la Educación Superior y la búsqueda del cambio social y el desarrollo. Conferencia Mundial sobre la Educación Superior. Retrieved from: http://www.me.gov.ar/spu/documentos/Declaracion_ conferencia_Mundial_de_Educacion_Superior_2009.pdf(consulted 11/10/2013).

Universidad de La Habana (2010). Plan de Desarrollo para los adiestrados de Nivel Superior de la Universidad de La Habana. Cursos 2009-2010. 
Vallaeys, F. (s/f). Hacia la construcción de indicadores de responsabilidad social universitaria. http://blog. pucp.edu.pe/eticaRSU (consulted 03/2012).

Vallaeys, F. (s/f). Breve marco teórico de Responsabilidad Social Universitaria. http:// www.iadb.org/etica (consulted 03/2012).

Velázquez, E. (2012). La calidad del ingreso a la Educación Superior. Reto del sistema educacional cubano, La Habana, Palacio de las Convenciones.

Vera, L. J. et al. (2012). La docencia en el marco de la responsabilidad social universitaria. Opción, año 28, 68, 257-272. Retrieved from: http://www.redalyc.org/ revista.oa?id=310 (consulted 25/03/2014).

Zolezzi Ibarcena, L. (s/f). La responsabilidad social en la formación de los abogados. Revista Derecho PUCP, 65, 251-261.

\section{Riassunto}

In questo articolo si analizzano le principali esigenze che affrontano professori e studenti nel momento attuale nel contesto della educazione superiore cubana. Questi due attori del processo educativo devono assumere la loro attivitá con un alto senso di responsabilitá e impegno sociale per le implicazioni nelle trasformazioni sociali ed economiche che si stanno sviluppando nel Paese. Il miglioramento della formazione di studenti e professori è oggetto di indagine in tutto il mondo: attualmente gli studi della Responsabilità Sociale Universitaria (RSU) stanno dimostrando la loro validità nel conseguimento di tanto importanti propositi. In questo senso, il Gruppo di Interazione Sociale Universitaria, del Centro di Studi per il Perfezionamento dell'Educazione Superiore, dell'Università di L'Avana sta sviluppando una ricerca sulla formazione RSU con studenti e professori come contributo per realizzare un modello ideale del corpo studentesco e professorale. Tale processo formativo è volto a promuovere lo sviluppo sociale, etico ed emotivo per il benessere personale e sociale.

Parole chiave: Educazione superiore cubana, Formazione continua, Formazione integrale, Modello ideale del corpo studentesco e professorale, Responsabilità Sociale Universitaria.

How to cite this Paper: Ojalvo Mitrany, V., \& Curiel Peón, L. (2015). La formación integral del estudiante y la formación continua de los profesores en la Educación Superior cubana: el papel de la Responsabilidad Social Universitaria (RSU) en su consecución [Integral formation of student and continuous formation of professors in Cuban Higher Education: A role for University Social Responsibility (USR)]. Journal of Educational, Cultural and Psychological Studies, 12, 257-282. doi: 10.7358/ecps-2015-012-ojal 